\title{
BEAM OPTICS OF A 10-CM DIAMETER HIGH CURRENT HEAVY ION DIODE *
}

\author{
J.W. Kwan, J.L. Vay, F.M. Bieniosek, Lawrence Berkeley National Laboratory; \\ E. Halaxa, G. Westenskow, Lawrence Livermore National Laboratory; I. Haber, Univ. of Maryland
}

\section{Abstract}

Typically a large diameter surface ionization source is used to produce > $0.5 \mathrm{~A} \mathrm{~K}^{+}$current with emittance $<1 \pi$-mm-mrad for heavy ion fusion experiments. So far we have observed aberrations that are slightly different from those predicted by computer simulations. We have now set up an experiment to study in detail the beam optics of such a large diameter ion diode and to benchmark the simulation code.

\section{INTRODUCTION}

Heavy ion driven inertial fusion (HIF) requires about 3$7 \mathrm{MJ}$ to achieve ignition with a D-T target [1]. At 2-4 $\mathrm{GeV}$ ion kinetic energy, the corresponding beam charge is $\sim 1 \mathrm{mC}$. Induction linacs can accelerate and compress these beams from $\sim 10 \mu \mathrm{s}$ at injection to $\sim 300 \mathrm{~ns}$ by the end of the driver and further drift compress the duration down to $\sim 10 \mathrm{~ns}$ at the target. The total beam current from the ion source is $\sim 50-100 \mathrm{~A}$. In order to overcome the space charge problem associated with high current heavy ion beams, an HIF driver is usually designed to contain an array of $\mathrm{N} \sim 100$ parallel ion beam channels at $\sim 0.5 \mathrm{~A}$ each.

In order to focus the ion beams onto a mm-size fusion target the beam emittance must be small, thus HIF requires beams with both large current and high brightness. Since the beam brightness is proportional to $\mathrm{J} / \mathrm{T}$, where $\mathrm{J}$ is the current density and $\mathrm{T}$ is the effective ion temperature, high brightness demands either high current density and/or low effective ion temperature. Furthermore, a heavy ion injector must have an adequate low energy beam transport (LEBT) system that can handle the severe space charge force. The LEBT often limits the maximum current density in the injector and therefore dictates the type of ion source that can be used.

According to high voltage breakdown and ChildLangmuir space-charge flow scalings, the current density of a diode decreases as the beam current increases [2]. Thus producing large current and high brightness from a single large aperture implies low ion temperature because the current density must be low. This condition can be met by a surface ionization sources because their typical effective ion temperature is $<1 \mathrm{eV}$ and the solid emitter surface provides a way to design large diameter beam optics.

Although heavy ions such as $\mathrm{Cs}^{+}$are ultimately needed

\footnotetext{
**This work is supported by the Office of Fusion Energy Science, US DOE under contract No. DE-AC03-76SF00098 (LBNL) and W-7405ENG-48 (LLNL). Email: jwkwan@lbl.gov
}

for fusion drivers, lighter ions such as $\mathrm{K}^{+}$can be useful in the near future because they provide an opportunity to do experiments at high ion velocities on medium length accelerator facilities during the early development phases.

Figure 1 shows the "2-MV" injector that was developed for the previous ILSE project [3] which consists of a 750 $\mathrm{kV}$ triode followed by an electrostatic quadruple (ESQ) section. Recently the ion source and extraction gap was modified in order to improve the beam optics for meeting the requirements of the High Current Transport (HCX) experiment [4]. So far the experimental results from the HCX experiments have shown qualitative agreement with the computer simulation. The discrepancy in beam current and in beam optics between the measurement and simulation predictions were significant enough to cause uncertainty in future beamline designs. This is especially true if the design relies heavily on "end-to-end simulation" or the experiment requires a high degree of phase space control accuracy.

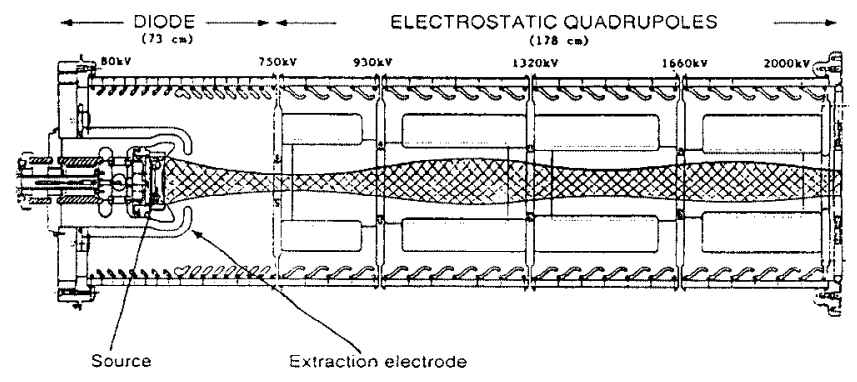

Fig. 1: Schematic Diagram of the 2-MV injector.

At present, our strategy is to use a two-pronged approach for the injector program [2]. On one hand we are exploring a new approach to built compact injectors, that are suitable for multiple beams HIF drivers, using an array of high current density beamlets. On the other hand, we continue to improve the large diameter surface source so it can provide a single-beam injector for near-term HIF projects such as the Integrated Beam Experiment (IBX). Recently we have mastered the techniques to fabricate large diameter alumino-silicate sources with a uniform emitting surface [5].

The main objective of the experiment described in this paper is to study how to produce a high quality ion beam from a large diameter surface ionization source. By carefully comparing the results from experiment against that from computer simulation, we will benchmark the simulation code(s) and also determine if the necessary physics are included in the simulations. 


\section{RECENT DEVELOPMENT}

Previous injector experiment with the ILSE project have produced up to $0.8 \mathrm{~A}^{\text {of }} \mathrm{K}^{+}$ion beam at $2.0 \mathrm{MeV}$ by using a $17-\mathrm{cm}$ diameter ion source [3]. However the beam profile was found to be hollow with significant current density non-uniformity. Since spherical aberration occurs near the edge of the emitter and is more pronounced when the source diameter is large, we reduced the ion source diameter down to $10 \mathrm{~cm}$, and modified the extraction electrode. The result was a lower beam current at $0.6 \mathrm{~A}$, but the beam optics was significantly improved $[4,6]$.

Figure 2a shows a Kapton film image of the beam spot at the injector exit (accumulated exposure of 100 beam pulses). Previous calibration confirmed that the darkening on a Kapton film was proportional to beam exposure at the 2-MV energy range [7]. Since the image was timeintegrated over the entire pulse length, it also recorded (superimposed) the beam head and tail that were responsible for the complicated pattern inside the ellipse. In comparison to the corresponding time-integrated image generated by computer simulation, as shown in Fig. 2b, we found a $\sim 15 \%$ discrepancy in the rms envelope size and a $\sim 20 \%$ discrepancy in the beam current. We found the discrepancy was minimized if the extraction voltage used in the simulation was empirically re-calibrated from the measured value. This finding suggested that there could be a possible error in the extraction voltage measurement but so far we were not able to confirm that assumption.
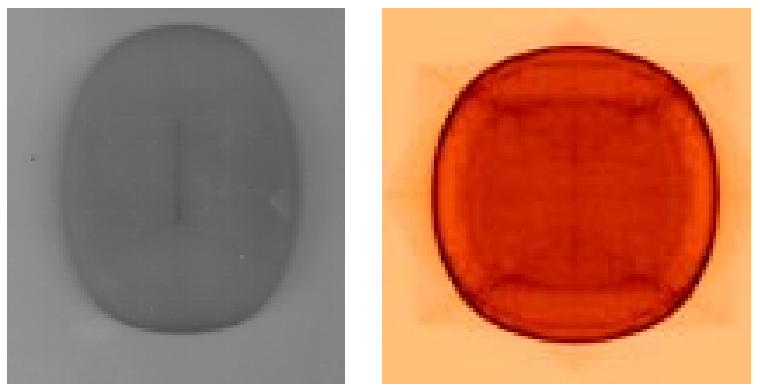

Fig. 2a. Kapton image of beam spot at the injector exit. $2 b$. Images obtained from time-dependent simulation.

\section{COMPUTER SIMULATIONS}

In order to minimize current density non-uniformity and aberrations, the extraction diode must be designed by using reliable computer codes. In the HIF program we have used both EGUN and WARP-3D. Figure 3 shows the result from WARP-3D simulation of a beam extracted from a curved emitter $(10 \mathrm{~cm}$ diameter). Figure $4 \mathrm{a}$ is the corresponding current density profiles at two axial locations and Fig. $4 \mathrm{~b}$ is the results from a flat emitter. In comparison, the flat emitter produces a more uniform current density beam.

When comparing results between different simulation codes, and also results from the same code but using different mesh sizes or numerical convergent criteria, we often found that the fine details of the beam profile may vary. For example, the "ripples" on the beam profile in Fig. 4a should not be taken too seriously and the absolute height of the spikes at the beam edge can be very sensitive to small variation. This kind of error can result in up to $10 \%$ uncertainty in the total beam current.

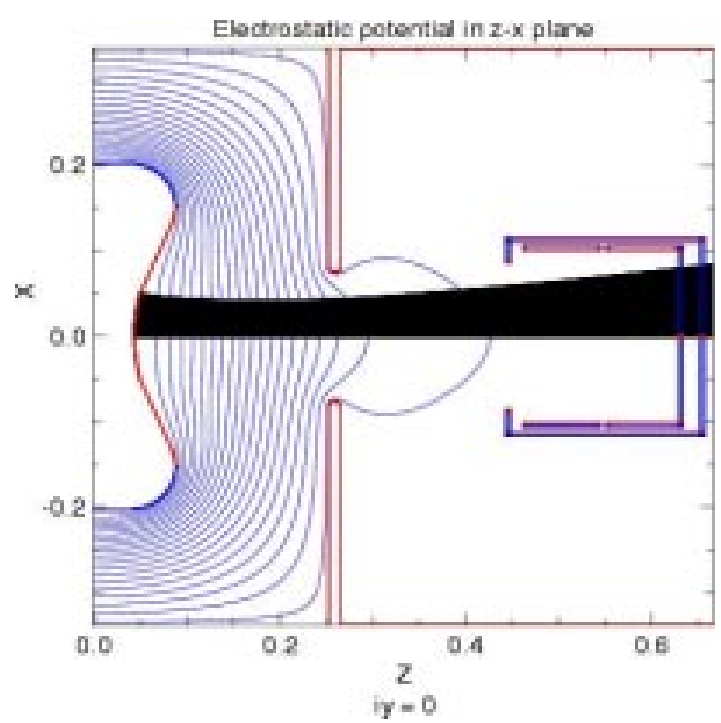

Fig. 3: WARP-3D simulation of a diode producing an ion beam entering a Faraday cup (only trajectories on upper-half plane are shown).
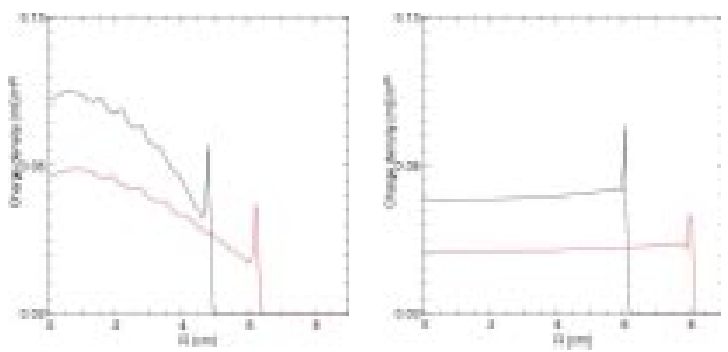

Fig. 4a Current density profiles of a curved source at $\mathrm{z}=0.3 \mathrm{~m}$ and $\mathrm{z}=0.45 \mathrm{~m}$. Fig. $4 \mathrm{~b}$ : corresponding profiles for a flat source.

Part of the errors may be due to mesh size being too coarse at critical regions such as the gap between the emitter edge and the tip of the Pierce electrode. An effective way to deal with this problem is to use adaptive mesh [8]. Another possible source of error is the method used to determine the local current density at the emitting surface. Each code seems to have its own unique way of dealing with this boundary condition.

Aside from numerical errors, the simulation can still be inaccurate if the physics is incomplete. For example, the code may ignore effects such as secondary electrons, alignment errors, ion temperature, work function and roughness of the emitting surface. In our case, HIF beams are both high current and low emittance. With high current, the space charge effect is severe, so the secondary 
electron problem can be significant. With low emittance, the effective ion temperature is only a small fraction of an $\mathrm{eV}$, therefore the work function and surface roughness can be important.

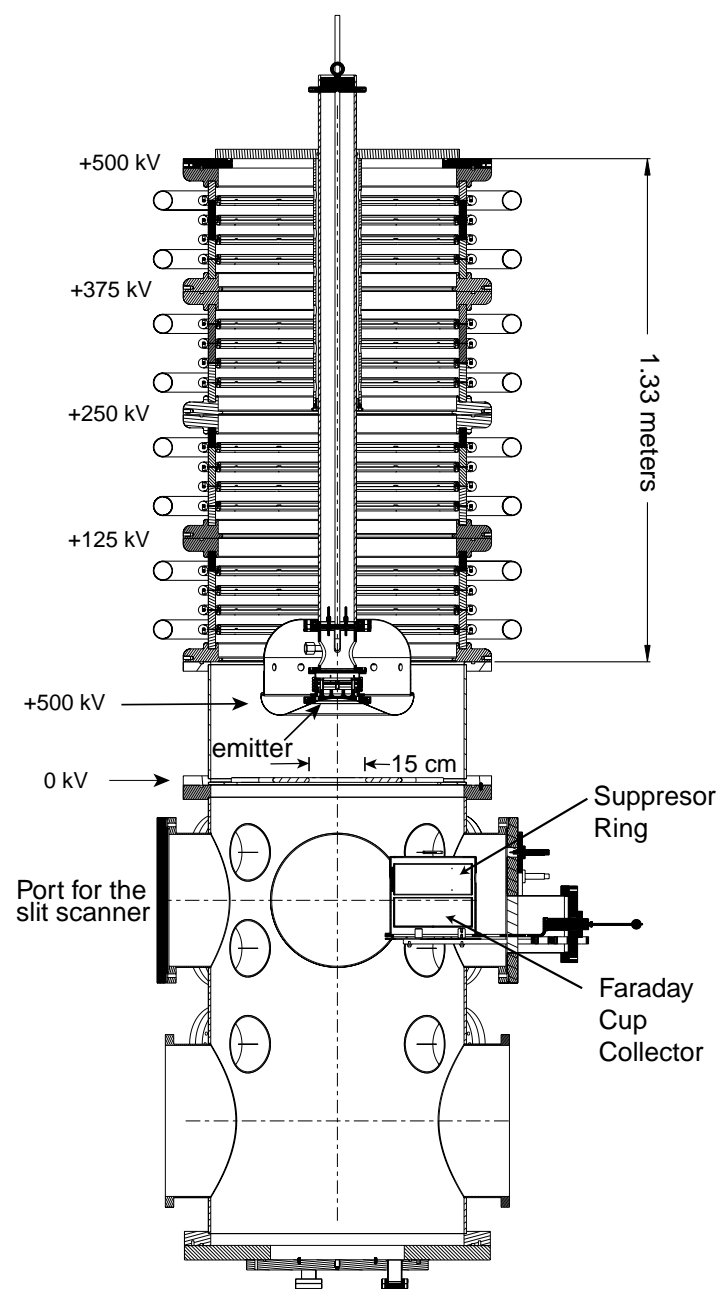

Fig. 5: Schematic diagram of a $10-\mathrm{cm}$ alumino-silicate ion source/diode experiment showing the Faraday cup in the retracted position.

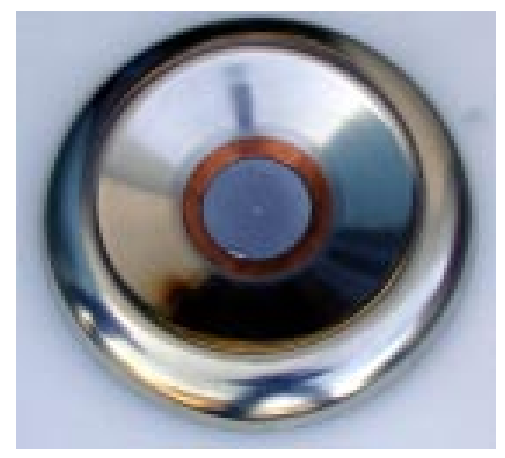

Fig. 6: Photograph of the $10-\mathrm{cm}$ diameter aluminosilicate ion source mounted on a Pierce electrode.
In electrostatic devices, the charged particle trajectories remain similar if $\mathbf{J} \sim \mathrm{V}^{1.5} / \mathrm{L}^{2}$ where $\mathrm{J}$ is the current density, $\mathrm{V}$ is the voltage, and $\mathrm{L}$ is the characteristic length. Since the ion temperature, the work function, and the surface roughness are fixed parameters determined by the ion source surface condition, they do not scale according to the diode voltage or the source diameter. Likewise, production of secondary electrons increases rapidly with the beam potential. The important point here is that while reduced size experiment can be useful for proof of principle purpose, only real size experiment can study the "dirty physics" which is critical for a practical driver.

\section{NEW EXPERIMENTAL SETUP}

Figure 5 shows the schematic diagram of a new experiment at LLNL dedicated to study the large-diameter ion source and extraction diode problem. Figure 6 is a picture of the $10-\mathrm{cm}$ source mounted on a large Pierce electrode. The test stand can provide up to $500 \mathrm{kV}$ and 20 $\mathrm{ms}$ of diode voltage. Beam diagnostics will include Faraday cup, beam spot imaging, slit-scanner and pepperpot for emittance measurements.

Our goal is to use the experimental results from this experiment for benchmarking the computer simulation codes. Once we have a reliable code, with all the necessary physics included, we hope to improve the beam optics and therefore enhance our ability to observe subtleties in future large-aperture-fill HIF experiments. At the time of writing this paper, the experiment has just begun commissioning. The ion source was heated to $1000 \mathrm{deg} \mathrm{C}$ and we saw no relative movement between the ion source and the Pierce electrode that could cause alignment errors. We expect to publish the experimental results, in comparison with computer simulations, by the end of this year.

\section{REFERENCES}

[1] D. A. Callahan-Miller, M. Tabak, Nuclear Fusion 39, 1547 (1999).

[2] J.W. Kwan, Rev. Sci. Instrum, 71(2), p807, (2000).

[3] S. Yu, et al, Fusion Engineering and Design, 32-33, p309, (1996)

[4] J. W. Kwan, F. M. Bieniosek, E. Henestroza, L. Prost and P. Seidl, Laser and Particle Beams, 20, p 441-445, (2002).

[5] D. Baca, E. Chacon-Golcher, J. W. Kwan, J. K. Wu, see this proceeding.

[6] F. M. Bieniosek, E. Henestroza, and J.W. Kwan, Rev. Sci. Instrum, 73, p1042, (2002)

[7] F. M. Bieniosek, J.W. Kwan, L. Prost, and P. Seidl, to be published in Rev. Sci. Instrum. August, (2002). Kapton calibration

[8] J.-L. Vay, P. Colella, P. McCorquodale, B. Van Straalen, A. Friedman, and D.P. Grote, Laser and Particle Beams, 20, p569-575, 2002 